\title{
INTRAOCULAR MALIGNANT MELANOMAS PRESENTING WITH ORBITAL INFLAMMATION
}

\author{
GEOFFREY E. ROSE ${ }^{1}$, H. BING HOH ${ }^{2}$, RICHARD A. HARRAD ${ }^{2}$ and JOHN L. HUNGERFORD ${ }^{1}$ \\ London and Bristol
}

\begin{abstract}
SUMMARY
Symptoms and signs of orbital inflammation may be prominent features in the presentation of choroidal malignant melanoma, even when the tumour is solely intraocular. Three cases with this clinical presentation are described.
\end{abstract}

Choroidal malignant melanoma is the commonest intraocular malignancy, and extraocular extension of the tumour can occur in neglected cases. Extraocular extension is often accompanied by proptosis, restriction of ocular movements and signs of orbital inflammation. Orbital inflammation in the absence of extraocular tumour extension has been described only rarely ${ }^{1-3}$ and the importance of this serious diagnosis in the differential diagnosis of orbital cellulitis has not been stressed.

Three cases of solely intraocular choroidal malignant melanoma are described, in all of which there were prominent clinical features of orbital inflammation.

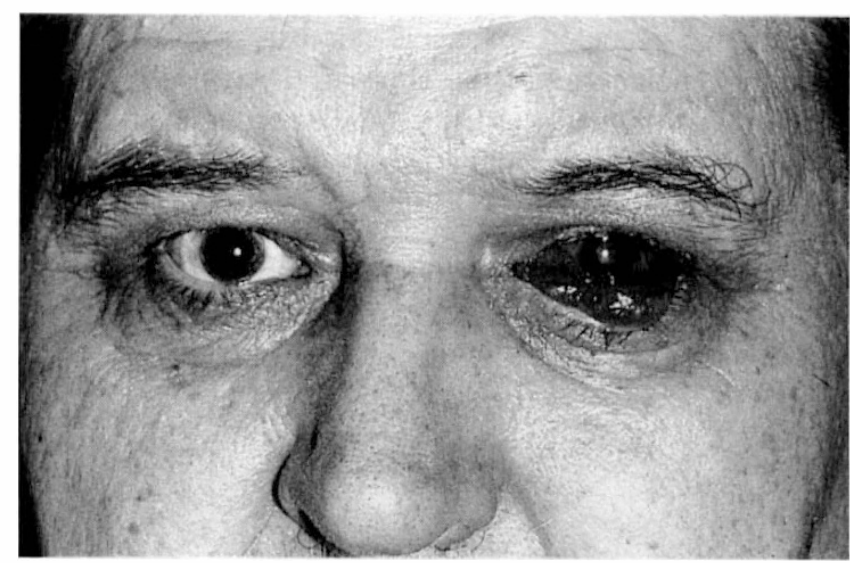

Fig. 1. Case 1. A 63-year-old man presenting with a 3-week history of blind and painful left eye, with $3 \mathrm{~mm}$ axial proptosis and limited ocular ductions.

From: 'Moorfields Eye Hospital, London; ${ }^{2}$ Bristol Eye Hospital, Bristol, UK.

Correspondence to: Mr. G. E. Rose, MS, FRCS, FCOphth, Moorfields Eye Hospital, City Road, London EC1V 2PD, UK.

\section{CASE REPORTS}

\section{Case 1}

A 63-year-old man presented to Moorfields Eye Hospital with a 3-week history of painful, red and swollen left eyelids, worse for the 2 days prior to presentation; the patient had declined earlier referral to hospital by his family practitioner. During this period the patient had had an influenza-like illness and claimed that his left vision had declined rapidly.

The right eye was normal (Snellen acuity 6/6) but the left eye had no perception of light, with a dense left relative afferent pupillary defect. There was $3 \mathrm{~mm}$ of left axial proptosis and a restriction of all ductions on that side. The left eyelids were red and swollen, marked conjunctival chemosis was present and there was prolapse of the inferior conjunctival fornix (Fig. 1). The left eye had corneal oedema, a very shallow anterior chamber, marked iris atrophy with a semi-dilated pupil, a mature cataract and an intraocular pressure of $45 \mathrm{mmHg}$.

B-mode ultrasonography suggested a bilobular mass occupying most of the posterior segment of the left globe, with no definitive evidence of extraocular extension. The proptosis, soft-tissue swelling of the eyelids and a left intraocular mass were shown on CT (Fig. 2).

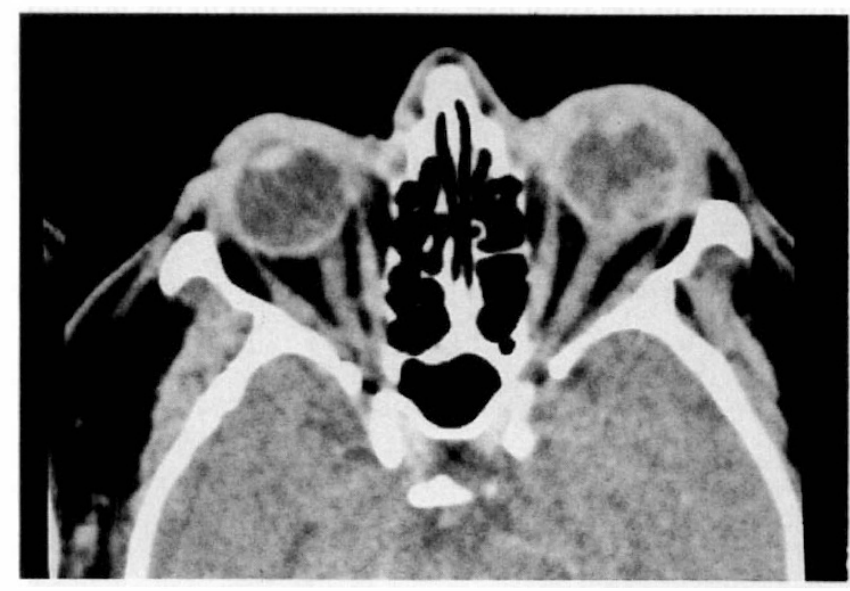

Fig. 2. Case 1.AxialCT scan showing soft-tissue swelling and proptosis secondary to intraocular melanoma. 


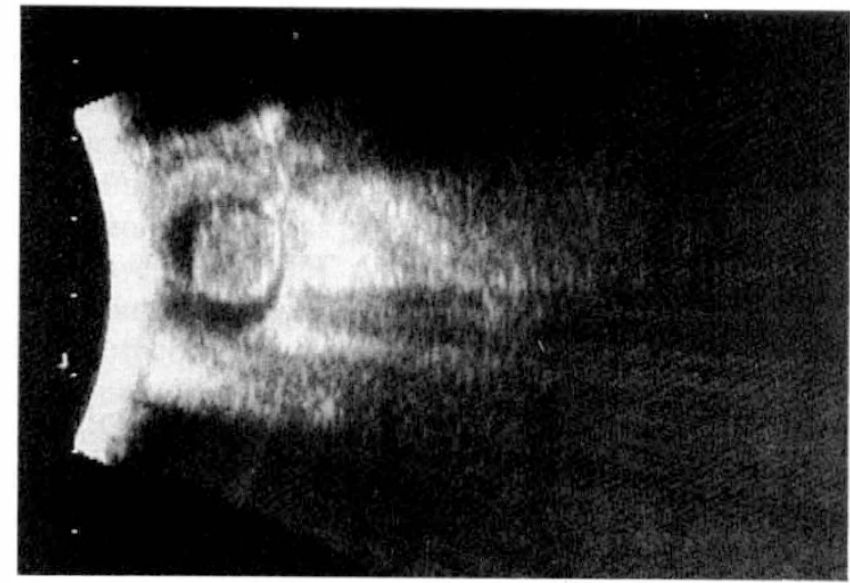

Fig. 3. Case 2. B-mode ultrasonography showing a solid posterolateral intraocular mass.

High-dose systemic steroids were administered to suppress periocular inflammation prior to surgical exploration; subsequent enucleation and histopathological examination demonstrated a solely intraocular choroidal malignant melanoma. The tumour was of spindle cell morphology and extended through Bruch's membrane into the subretinal space; minimal local extension of the tumour into underlying scleral lamellae was not accompanied by any evidence of extrascleral extension or tumour necrosis. Iris infarction and cataract were present and the drainage angles were occluded by fibrovascular membranes. Despite systemic steroids for 3 weeks prior to enucleation, there was a marked subconjunctival reaction with necrosis, oedema, episcleritis and corneal vascularisation. The appearances suggested anterior segment ischaemia and inflammation, possibly due to vascular occlusion in the territory of the posterior ciliary arteries.

\section{Case 2}

The patient, a 41-year-old man, presented to the Accident and Emergency Department at Bristol Eye Hospital with a 1-day history of painful, red right eye with systemic malaise and vomiting. The acuity was perception of hand movements in the right eye and Snellen 6/6 in the normal left eye. Corneal oedema was present on the right, with a

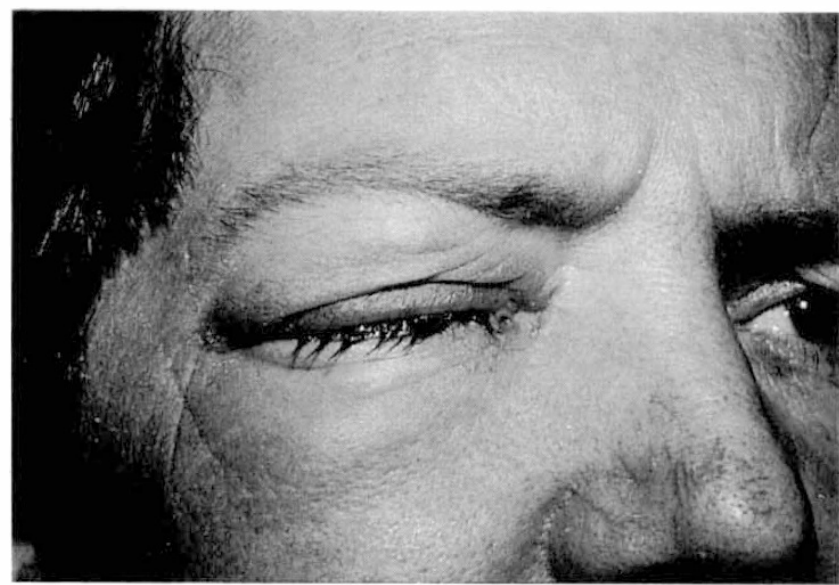

Fig. 4. Case 2. Right periocular inflammation causing $3 \mathrm{~mm}$ axial proptosis and reduced ocular ductions.

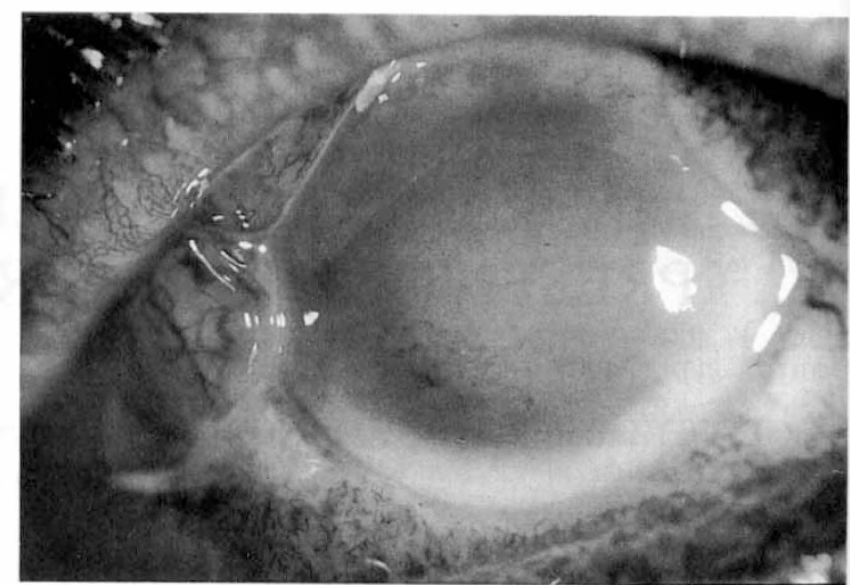

Fig. 5. Case 2. Intracameral deposits of melanin in the presence of intraocular and periocular inflammation.

shallow anterior chamber, rubeosis, a semi-dilated pupil, an intraocular pressure of $43 \mathrm{mmHg}$ and an inferolateral bullous retinal detachment.

B-mode ultrasonography revealed a large intraocular mass lying posterolaterally in the right eye (Fig. 3). Treatment with systemic acetazolamide and topical timolol was commenced.

Whilst being further investigated during the 3 days after presentation, the right eye became red and swollen and developed $3 \mathrm{~mm}$ axial proptosis with conjunctival chemosis and restriction of all ocular ductions (Fig. 4). The acuity deteriorated to no perception of light, anterior chamber depth decreased further and intracameral melanin deposits were evident (Fig. 5). A mild neutrophilia was present, although the patient remained well systemically. CT confirmed an intraocular mass, with proptosis and increased soft tissues in the right orbit and eyelids (Fig. 6).

Right enucleation was performed and histopathological examination revealed extensive necrosis within a large malignant melanoma of the choroid. There was no surgical or histological evidence of extrascleral extension and biopsies of intraorbital fat showed only inflammatory cell infiltration.

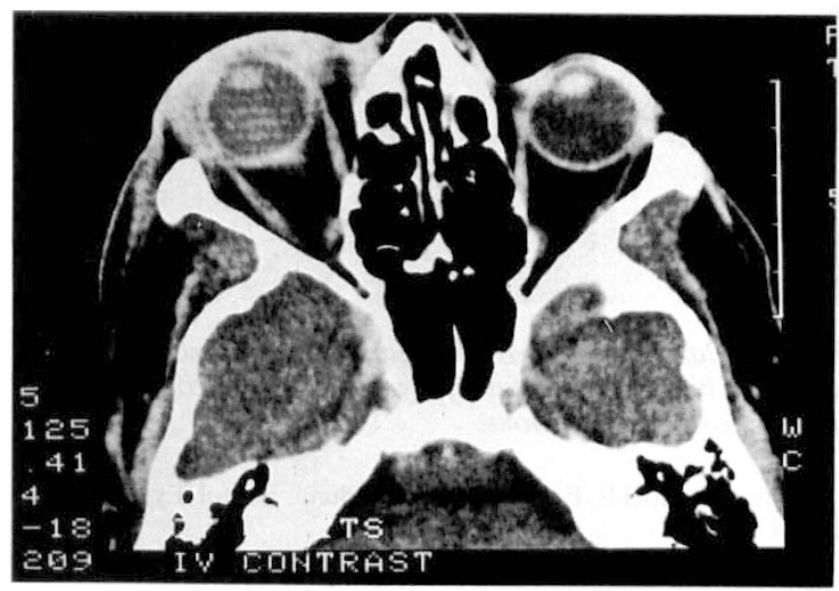

Fig. 6. Case 2. Axial CT scan showing soft-tissue swelling and proptosis secondary to intraocular melanoma. 


\section{Case 3}

A 48-year-old man presented to the Accident and Emergency Department at Moorfields Eye Hospital with a 1-day history of red and painful left eyelids, blurring of vision and severe periorbital pain radiating across his forehead.

The patient had visual acuities of Snellen $6 / 6$ in the right eye and $6 / 18$ in the left, with a mild left relative afferent pupillary defect. There was swelling of the left eyelids, with marked ciliary injection and conjunctival chemosis; the left globe was not displaced and eye movements appeared to be normal. Severe panuveitis was present, with a $1 \mathrm{~mm}$ neoplastic hypopyon, rubeosis, an intraocular pressure of $27 \mathrm{mmHg}$ and a dark tumour mass situated medially in the area of the ciliary body.

Whilst the patient was awaiting enucleation the left acuity deteriorated to perception of hand movements and the clinical signs increased. Enucleation confirmed a largely necrotic spindle-cell malignant melanoma arising in the pars ciliaris; scleral, episcleral and intraocular inflammation were present, but there was no evidence of extrascleral extension of the tumour.

\section{DISCUSSION}

A large number of choroidal melanomas are asymptomatic, often being discovered by optometrists during routine eye testing. Symptomatic intraocular tumours, however, generally present with disturbance of visual functions, either acuity or field, or with symptoms of intraocular inflammation, such as pain or ocular redness.

Enlargement of the tumour within the posterior segment of the globe may cause anterior displacement of the iris and lens with shallowing of the anterior chamber, as seen in two of the three cases presented. An impaired outflow of aqueous humour - due to rubeosis (cases 2 and 3 ), inflammatory or tumour cells obstructing the trabecular meshwork (case 3) or acute closure of the drainage angle (cases 1 and 2) - may lead to a rise in intraocular pressure or to frank acute angle closure glaucoma.

Where a patient may have failed to appreciate preceding symptoms of visual failure - loss of either visual field or acuity - the initial symptoms may be those of a severe acute inflammation. This inflammation may result from a neovascular uveitis, from acute angle closure glaucoma or from tumour necrosis; ${ }^{2}$ extensive tumour necrosis was present in cases 2 and 3 . Tumour necrosis might be the sole cause of the uveitis, or the uveitis and the necrosis might be coincident manifestations of an abnormal intraocular immune status.

Because some patients may be unaware of preceding symptoms, the possibility of a tumour within the posterior segment of the globe should be considered in all patients presenting with acute orbital inflammation.

The authors wish to thank Dr. Alison McCartney (London) and Dr. C. Collins (Bristol) for their reports of the tumour histopathologies.

\section{REFERENCES}

1. Fraser DJ Jr, Font RL. Ocular inflammation and haemorrhage as initial manifestations of uveal malignant melanoma: incidence and prognosis. Arch Ophthalmol 1979;97:1311-4.

2. Bujara K. Necrotic malignant melanomas of the choroid and ciliary body: a clinicopathological and statistical study. Graefes Arch Clin Exp Ophthalmol 1982;219:40-3.

3. Thatcher DB. Necrotic choroidal malignant melanoma presenting with severe inflammation. Surv Ophthalmol 1967; 12:247-53. 